\title{
Candidemia in Neonatal Intensive Care Units: Magnitude, Species Identification And Antifungal Susceptibility Pattern
}

\author{
Dr Suresh Kumar Chavhan, ${ }^{1}$ Dr Jeetam Singh Meena, ${ }^{2}$ Dr Kailash Kumar Meena ${ }^{3}$, \\ Dr Jitendra Kumar Meena ${ }^{4}$ \\ ${ }^{I}$ Resident, Department Of Paediatric Medicine, , SMS Medical College \& Hospital, Jaipur \\ ${ }^{2}$ Assistant Professor, Department Of Pediatric Medicine, SMS Medical College And Hospital, Jaipur \\ ${ }^{3}$ Senior Professor And Unit Head, Department Of Pediatric Medicine, SMS Medical College And Hospital, \\ Jaipur. \\ ${ }^{4}$ Senior Resident, Department Of Pediatric Medicine, SMS Medical College And Hospital, Jaipur. \\ Corresponding Author: Dr Jeetam Singh Meena
}

\begin{abstract}
Background:-Invasive fungal sepsis contributing significant neonatal mortality and in last two decade changed in growing species and sensitivity pattern, this hospital base prospective observational study with aims to find magnitude of candidemia and drug susceptibility pattern of candida species in neonatal intensive care units at tertiary level center.

Methods: All Preterm and full term infants with risk factors for fungal infection admitted in neonatal intensive care unit (167) of Zenana and Mahila chikitsalaya during the study period (May 2014-April 2015) were included. The candida isolates were identified with automated Vitek-2 compact system of Biomeriux, Germoney using the YST ID cards and their antifungal susceptibility was performed using AST YS 06 Cards.

Results: A total of 20 (11.97\%) Candida isolates were found among 167 NICU patients. Candida albicans (35\%) was the most common isolate followed by Candida parapsilosis (30\%), Candida tropicalis (15\%), Candida krusei (15\%), and Candida glabrata (5\%). Among the 20 Candida isolates, resistance to the Fluconazole was 35\%, to Ketoconazole 25\%, to Miconazole 20\%, to Amphotericin-B 15\%, and to Caspofungin $5 \%$.

Conclusion: Increased incidences of candidemia along with emergence of Non-albicans Candida species are become an important healthcare issue worldwide. Therefore, the early and accurate identification of Candida spp along with susceptibility testing is very important.
\end{abstract}

Keywords: Antifungal susceptibility, Candidemia, Candida albicans, Non albicans candida

\section{Introduction}

Neonatal deaths account for $40 \%$ of deaths under the age of 5 years worldwide. Therefore, efforts to achieve the UN Millennium Development Goal 4 of reducing childhood mortality by two-thirds by 2015 are focused on reducing neonatal deaths in high-mortality countries ${ }^{1}$. Sepsis is the commonest cause of neonatal mortality; it is responsible for about $30-50 \%$ of the total neonatal deaths in developing countries ${ }^{2}$. The pathogens most often implicated in neonatal sepsis are bacteria, although with increasing survival of smaller, more immune-compromised preterm infants, the incidence of invasive fungal infection is increasing among Neonatal Intensive Care Unit (NICU) patients. After gram positive and gram negative bacteria, Candida species are the third most frequent cause of septicemia after 72 hours of life in neonates with estimated incidence of 1.6 to $9 \%$ among very low birth weight (VLBW) and 10 to $12 \%$ among extremely low birth weight (ELBW) neonates in neonatal intensive care units. Infections due to invasive fungal infections account for $15 \%$ of all blood stream infections (BSI) in the $\mathrm{NICU}^{3}$. Candida albicansis the most frequently recovered fungus, but in the last two decades the trend has changed with C. parapsilosis as the more prevalent organism. Aspergillusis second only to Candida as a cause of invasive fungal infections in neonates. Invasive candidemia is associated with a mortality of $38 \%$ and a crude mortality of 30\%-75\% ${ }^{4}$. This study is designed with primary objective to identify specific candida species and their drug sensitivity pattern that helps in diagnosis and effective management of candidiasis in neonatal intensive care unit.

\section{Material And Methods}

This is a hospital based prospective observational study carried out in neonatal intensive care units of Zenana and Mahila Chikitsalaya attached to SMS Medical College and Hospital, Jaipur after obtaining clearance from institute ethical committee. After obtaining written informed consent, 167 neonates admitted in NICU from May 2014 to April 2015 with inclusion criteria prematurity, lower birth weight, selected term neonates with risk factors like clinical evidence of sepsis, history of premature rupture of membrane, maternal history of symptoms of lower genital tract infection, maternal fever, gestational diabetes and requiring parenteral nutrition, 
mechanical ventilation more than 7 days, central venous catheter, $\mathrm{H} 2$ blocker therapy, and antibiotics more than 7 days were included. Full term neonates admitted in NICU with indication other than sepsis like metabolic disease, neonatal jaundice, congenital malformations were excluded. A thorough history, physical examination and routine investigations were done in every case and recorded in the pre-designed performa. Sample for blood cultures was taken in Brain Heart Infusion (BHI) bottle at the time of admission to NICU and weekly thereafter till discharge or death. The BHI bottle was incubated at $37^{\circ} \mathrm{C}$ in the laboratory and observed daily for visible growth. Subculture was performed after 48 hours and on 7 th day before being reported negative. If visible growth was observed earlier, they were sub cultured on primary inoculation media i.e. Blood agar (BA), MacConkey agar (MCA). Colonies of yeast on blood agar were identified by their smooth creamy colored appearance and Gram's staining. All isolates of candida were characterized and subjected to antifungal susceptibility testing using the Vitek-2 yeast YST ID and antimicrobial susceptibility test (AST) YS 06 cards as per to manufacturers (Biomeriux) instructions and results were evaluated ${ }^{5}$.

The Vitek-2 is an automated microbial identification system that provides highly accurate and reproducible results. Fresh subcultures of all the Candida isolates were obtained on sabouraud's dextrose agar (SDA) plates for identification and antifungal susceptibility testing using the Vitek 2 Compact, YST ID card, and AST YS 06 cards as per manufacturer's instructions. In AST YS 06 cards, antifungal susceptibility results based on interpretative guidelines recommended by Clinical Laboratory Standards Institute (CLSI) 2012 were obtained for fluconazole, ketoconazole, miconazole, amphotericin-B, and caspofungin.

\section{Statistical analysis}

Med Calc 12.2.1.0 version software was used for statistical calculation. Nominal and categorical data were summarized as proportion (\%). Chi-square test was used for analysis of nominal and categorical data. Odds ratio was calculated and $95 \%$ confidence interval was estimated. P-value $<0.05$ was taken as significant.

\section{Results}

In our study, out of 167 neonate enrolled, 43 (25.74\%) showed microbial growth in their blood culture. Among these $23(13.77 \%)$ and $20(11.97 \%)$ showed bacterial and candida blood stream colonization respectively in blood culture. Male preponderance was seen with male to female ratio 1.2:1. Majority of newborns was preterm $140(83.8 \%)$ \{Table I\}. Out of the 20 isolates of various candida species, the non albicans candida species were the predominant accounting for $13(65 \%)$ and the remaining $7(35 \%)$ isolate were of $C$. albicans $\{$ Table II $\}$. Maximum isolates were of $C$. albicans $(\mathrm{n}=7,35 \%)$, followed by $C$. parapsilosis $(\mathrm{n}=6$, $30 \%), C$. krusei $(\mathrm{n}=3,15 \%), C$. tropicalis $(\mathrm{n}=3,15 \%)$ and $C$. glabrata $(n=1.5 \%)\{$ Table III $\}$. Candida species are maximally resistant to fluconazole (35\%) whereas least to newer antifungal capsofungin (5\%) \{Table IV\}.

Table I: Baseline characteristics of the study participants

\begin{tabular}{|c|c|c|}
\hline \multicolumn{2}{|c|}{ Variables } & Number (n=167) \\
\hline \multirow{2}{*}{ Sex wise distribution } & Male & 92 \\
\cline { 2 - 3 } & Female & 75 \\
\hline \multirow{4}{*}{$\begin{array}{c}\text { Gestational age wise } \\
\text { distribution }\end{array}$} & $<28$ week & 14 \\
\cline { 2 - 3 } & & 68 \\
\cline { 2 - 3 } & 28 to <32 week & 58 \\
\cline { 2 - 3 } & 32 to <37 week & 26 \\
\cline { 2 - 3 } & 37 to 42 week & 1 \\
\cline { 2 - 3 } & $>42$ week & 64 \\
\hline \multirow{3}{*}{ Weight wise distribution } & ELBW & 60 \\
\cline { 2 - 3 } & VLBW & 30 \\
\cline { 2 - 3 } & LBW & 12 \\
\cline { 2 - 3 } & Normal & \\
\hline
\end{tabular}

Table II: Distribution of candida albicans and Non albicans Species

\begin{tabular}{|c|c|c|}
\hline Species & Number & Percentage (\%) \\
\hline Candida albicans & 07 & $35 \%$ \\
\hline Non albicans candida & 13 & $65 \%$ \\
\hline Total & 20 & $100 \%$ \\
\hline
\end{tabular}


Table III: Distribution of Candida according to species by Vitek-2 yeast identification

\begin{tabular}{|c|c|c|}
\hline Candida species & Number of isolates & Percent \\
\hline Candida albicans & 07 & $35 \%$ \\
\hline Candida parapsilosis & 06 & $30 \%$ \\
\hline Candida krusei & 03 & $15 \%$ \\
\hline Candida tropicalis & 03 & $15 \%$ \\
\hline Candida glabrata & 01 & $05 \%$ \\
\hline Total & 20 & $100 \%$ \\
\hline
\end{tabular}

Table IV: Antifungal Resistance pattern among candida isolates

\begin{tabular}{|c|c|c|c|c|}
\hline \multirow{2}{*}{$\begin{array}{c}\text { Candida species } \\
(\mathbf{n})\end{array}$} & \multicolumn{4}{|c|}{ Resistance } \\
\cline { 2 - 5 } & Fluconazole & Ketoconazole & Miconazole & Amphotericin-B \\
\hline C. albicans(7) & $14.2 \%$ & $20.56 \%$ & $28.57 \%$ & $14.2 \%$ \\
\hline C.parapsilosis(6) & $33.33 \%$ & $16.66 \%$ & $0 \%$ & $16.66 \%$ \\
\hline C. tropicalis(3) & $33.33 \%$ & $33.33 \%$ & $0 \%$ & $0 \%$ \\
\hline C. krusei(3) & $66.66 \%$ & $0 \%$ & $33.33 \%$ & $0 \%$ \\
\hline C. glabrata(1) & $100 \%$ & $100 \%$ & $100 \%$ & $100 \%$ \\
\hline Total (20) & $35 \%$ & $25 \%$ & $20 \%$ & $15 \%$ \\
\hline
\end{tabular}

\section{Discussion}

Fungal septicaemia is a common occurrence in Neonatal Intensive Care Unit patients. Prolonged hospital stay of premature babies, intense therapy, invasive procedures, central venous catheters, total parenteral nutrition, and various other risk factors leads to blood stream infection (BSI) leading to sepsis with various bacterial and fungal agents. Since various species differ in their antifungal susceptibility pattern, antibiotic susceptibility testing also done.

There is wide variation in the reported prevalence and incidence of fungal septicaemia in neonates admitted in NICU, ranging from 2.6 to $16.7 \%$. The incidence of candidemia in our study were $11.97 \%$ that is similar to study conducted by Agrawal et al and Rani et al showing isolation rate of $13.6 \%$ and $11 \%$ respectively ${ }^{5,6}$. Prevalence of candida species in blood stream infection has increased worldwide in the last decades. In the last few years occurrence of non albicans candida species are steadily increasing. In our study candida species other than C. Albicans were found to be leading cause of candida, BSI in neonates admitted in NICU accounted $13(65 \%)$ of total 20 candida isolates cases. Maria et al (1994) ${ }^{7}$ in his study also reported that C. Albicans was isolated in $90 \%$ of positive culture and remaining $10 \%$ isolate were of C. Tropicalis. C. Albicans was the leading causative organism isolated in $7(35 \%)$ of all cases diagnosed $(\mathrm{n}=20$ isolates), followed by C. parapsilosis $6(30 \%)$, C. tropicalis $3(15 \%)$, C. krusei $3(15 \%)$ and C. Glabrata $1(05 \%)$. ShabinaAriff et al $(1996)^{8}$ reported $55 \%$ of all cases diagnosed $(n=49$ isolates) were of C. albicans, followed by C. tropicalis $(21 \%)$, and C. glabrata (9\%). Although fluconazole still remain a safe and effective choice for the treatment of candidemia, there are increasing trends of fluconazole resistance has been reported in many studies as $37.5 \%$ by Gupta et $\mathrm{al}^{9}, 36 \%$ by Kothari et $\mathrm{al}^{10}, 25 \%$ by Adhikari et $\mathrm{al}^{11}$ and $11.7 \%$ by Xess et al ${ }^{12}$. In the present study $35 \%$ of candida was resistance to fluconazole. Non albicans candida species, especially $\mathrm{C}$. glabrata and C. krusei were found more resistant to azoles, particularly fluconazole, than C. albicans. $92.31 \%$ Candida albicans isolates were susceptible to fluconazole and itraconazole, while only $21.43 \%$ of C. krusei and $26.32 \%$ of C.glabrata isolates were susceptible to fluconazole. These two species were also only $21.43 \%$ and $36.84 \%$ susceptible to itraconazole respectively. The changing spectrum of candida species causing candidemia from C. albicans to Non albicans candida esp. C. glabrata and C. krusei is responsible for fluconazole resistance. In present study resistance to Amphotericin-B among all candida isolates was $15 \%$. Similar results were reported by Jaswinder K Oberoi et $\mathrm{al}^{13} 10.4 \%$ and $\mathrm{M}$ Bhatt et $\mathrm{al}^{14} 8 \%$.

Although Amphotericin-B has a rapid cidal action against most strains of candida species (esp. C.albicans), it is not the first choice due to associated nephrotoxicity and the newer lipid formulation having a better cidal effect profile. However in the developing country like India, fluconazole is the most widely drug of choice for treatment of candidemia due to its low cost, higher bioavailability along with intravenous (IV) formulation. In present study $5 \%$ isolates were resistant to caspofungin which correlated with the study of Michael A.P faller et $\mathrm{al}^{15}$ and Maria Teresa Montagna et $\mathrm{al}^{16}$ found $1.9 \%$ and $3.8 \%$ resistance respectively. Jaswinder $\mathrm{K} \mathrm{O}$ et $\mathrm{al}^{13}$ reported $100 \%$ sensitivity to caspofungin in his study. There was only one isolate of $\mathrm{C}$. glabrata which is resistant to fluconazole, ketokonazole, miconazole and amphotericin B while sensitive to caspofungin.

\section{Conclusion}

Increased incidences of candidemia along with emergence of Non-albicans Candida species are become an important healthcare issue worldwide. This study therefore emphasizes the need for a continuous surveillance of candidemia in the newborns admitted to intensive care units. Identification of candida up to 
species level and determination of their antifungal resistance pattern will help the clinician in the management of candidemia and to reduce the morbidity and mortality in these neonates.

\section{References}

[1]. ImtiazJehan, Hillary Harris, SohailSalat, AmnaZeb, NaushabaMobeen, Omrana Pasha et al. Neonatal mortality, risk factors and causes: a prospective population-based cohort study in urban Pakistan. Bulletin of the World Health Organization 2009; 87:130138.

[2]. Bang AT, Bang RA, Bactule SB, Reddy HM, Deshmukh MD. Effect of home-based neonatal care and management of sepsis on neonatal mortality, field trial in rural India. Lancet 1999; 354:1955-61.

[3]. Bendel CM. Colonization and epithelial adhesion in the pathogenesis of neonatal candidiasis. SeminPerinatol 2003 Oct; 27(5):357364.

[4]. Benjamin DK Jr, DeLong ER, Steinbach WJ, Cotton CM, Walsh TJ, Clark RH. Empirical therapy for neonatal candidemia in very low birth weight infants. Pediatrics 2003 Sep; 112(3 Pt 1):543-7.

[5]. Agarwal J, Bansal S, Malik GK, Jain A. Trends in nenonatal septicemia: emergence of non-albicans Candida. Indian Pediatr 2004; 41: 712-715.

[6]. Rani R, Mohpatra NP, Mehta G, Radhwa VS. Changing trends of candida species in neonatal septicaemia in a tertiary north Indian hospital. Ind J Medical Microbiol 2003; 21(1):56-58.

[7]. MaríaL. Avila-Aguero, Alejandro Canas-Coto, Rolando Ulloa-Gutierrez, Marco A. Caro, Braulio

[8]. ShabinaAriff, Ali Faisal Saleem, Sajid Bashir Soofi, ReemaSajjad. Clinical spectrum and outcomes of neonatal candidiasis in a tertiary care hospital in Karachi, Pakistan. J Infect DevCtries 2011; 5(3):216-223.

[9]. Gupta N, Mittal N, Sood P, Kumar S, Kaur R, Mathur MD. Candidemia in neonatal intensive care unit. Indian J PatholMicrobiol 2001; 44: 45-8.

[10]. Kothari A, Sagar V. Epidemiology of Candida Bloodstream Infections in a Tertiary Care Institute in India.Indian J Med Microbiol 2008; 27: 171-2.

[11]. Adhikary R, Joshi S. Species distribution and antifungal susceptibility of candidemia at a multi super specialty centre in Southern India. Indian J Med Microbiol 2011; 29: 309-11.

[12]. Xess I, Jain N, Hasan F, Mandal P, Banerjee U. Epidemiology of candidemia in a tertiary care centre of North India: 5-Year Study. Infection 2007; 35: 256-9.

[13]. Jaswinder Kaur Oberoi, Chand Wattal, NeerajGoel, ReenaRaveendran, S. Datta, Kamaljeet Prasad. Nonalbicans Candida species in blood stream infections in a tertiary care hospital at New Delhi, India. Indian J Med Res, 2012; 136: 997-1003.

[14]. M Bhatt, G Sarangi, BP Paty, D Mohapatra, N Chayani, A Mahapatra et al. Biofilm as a virulence marker in Candida species in nosocomial blood stream infection and its correlation with antifungal resistance. Indian Journal of Medical Microbiology, (2015) 33(Supplement 1): S112-14.

[15]. Michael A. Pfaller, Shawn A. Messer, Leah N. Woosley; Application of New CLSI Clinical Breakpoints and Epidemiological Cutoff Values for Characterization of Geographic and Temporal Trends of Antifungal Resistance. Echinocandin and Triazole Antifungal Susceptibility Profiles for Clinical Opportunistic Yeast and Mold Isolates Collected from 2010 to 2011 . Journal of Clinical Microbiology, 2013; 51(8): p. 2571-81.

[16]. Maria Teresa Montagna, GraziaLovero, CaterinaCoretti. Susceptibility to echinocandins of Candida spp.strains isolated in Italy assessed by European Committee for Antimicrobial Susceptibility Testing and Clinical Laboratory Standards Institute broth microdilution methods. BMC Microbiology 2015; 15:106. 